\title{
Thin-Shell Deployable Reflectors with Collapsible Stiffeners Part 1: Approach
}

\author{
Lin Tze Tan* \\ University College London, London, WC1E 6BT England, United Kingdom \\ and \\ Sergio Pellegrino ${ }^{\dagger}$ \\ University of Cambridge, Cambridge, CB2 1PZ England, United Kingdom
}

DOI: $10.2514 / 1.16320$

\begin{abstract}
Thin-shell deployable reflector structures that are folded elastically in a nearly inextensional mode have been recently realized, exploiting the recent availability of high-modulus, ultrathin composite materials. An inherent and significant limitation of this approach is that these structures remain "floppy" in their deployed configuration. This paper presents a general concept for increasing the deployed stiffness of such structures, through the addition of a collapsible edge stiffener around the rim of a reflector dish. An analytical expression of the frequency/stiffness related to the softest deformation mode of a thin-shell reflector structure is presented, both with and without the stiffener. During folding, the stiffener collapses elastically, and this behavior is facilitated by the introduction of suitable discontinuities within the stiffener, or between the dish and the stiffener. A detailed study of a range of different options is presented, and one particular scheme is selected and optimized. For a specific example, a stiffness increase by a factor of $\mathbf{3 1}$ and a fundamental frequency increase by a factor of $\mathbf{4}$ are achieved, with a mass increase of only $16 \%$.
\end{abstract}

\section{Nomenclature}

$A_{i}$
$B$
$b$
$c$
$D$
$d$
$d_{1}, d_{2}$
$E$
$F$
$F_{\text {final }}, F_{\max }$
$f$
$f_{i}$
$g_{1}, g_{2}$

$h$

$h_{0}$

$I_{R}, I_{T}$

$k, k_{0}$

$r, r_{s}$

$T$

$t$

$U$

V

$w$

$\gamma$

$\eta$

$\theta$

$=$ amplitude of mode $i$

$=$ thickness of rim

$=$

$=$ radius of coiling

$=$ plate bending stiffness

$=$ diameter

$=$ diameters of reflectors

Young's modulus

$=$ focal length of paraboloid

fundamental frequency

frequency of mode $(i-1)$

$=$ height of rim and tubular section

$=$ depth of rim

$=$ thickness of shell

$=$ strain energy

$=$ kinetic energy

$=$ width of stiffener

$=$ load slit angle

$=$ hinge slit angle
= final packaged force, maximum force

dimensionless parameters for calculation of natural frequencies of spherical shells

$=$ height of rectangular cross section

$=$ second moment of areas of rectangular section

$=$ initial stiffness, initial stiffness of configuration $\mathrm{O}$

$=$ radius, radius of midsurface of spherical shell

$=$ angle that defines a meridian of the shell
Received 27 February 2005; revision received 9 December 2005; accepted for publication 3 January 2006. Copyright $\odot 2006$ by Lin Tze Tan. Published by the American Institute of Aeronautics and Astronautics, Inc., with permission. Copies of this paper may be made for personal or internal use, on condition that the copier pay the $\$ 10.00$ per-copy fee to the Copyright Clearance Center, Inc., 222 Rosewood Drive, Danvers, MA 01923; include the code $\$ 10.00$ in correspondence with the CCC.

${ }^{*}$ Lecturer in Structural and Solid Mechanics, Department of Civil Engineering, Gower Street; 1.tan@ucl.ac.uk. Member of AIAA.

Professor of Structural Engineering, Department of Engineering, Trumpington Street; pellegrino@eng.cam.ac.uk. Associate Fellow of AIAA.

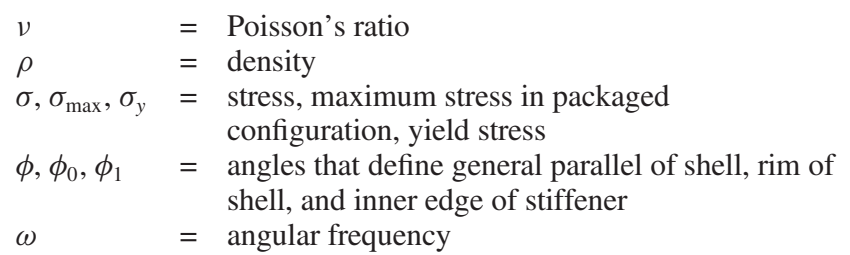

\section{Introduction}

$\mathbf{T}$ HIN-WALLED deployable structures have been used for a variety of spacecraft structures and, until recently, almost only in the form of singly curved shell structures. Notable examples include tubular metallic booms such as the storable tubular extendible mast [1] and the collapsible tube mast [2], and selflocking hinges consisting of short lengths of steel tape measure (tape springs) for solar arrays [3]. A feature that is common to all of these foldable structures is that they undergo a continuous process of inextensional elastic deformation that transforms the structure from its operational configuration into a compactly packaged configuration.

The recent availability of high-modulus, ultrathin composite materials has led to the realization of structures with greater functionality, through the use of complex three-dimensional shapes. Novel applications of this approach to deployable reflector structures have been proposed [4-6].

An example of particular relevance to the present paper is the spring back reflector $[7,8]$, an ultrathin flexible parabolic shell made from triaxially woven carbon-fiber reinforced plastic. The whole structure, shown in Fig. 1, is manufactured as a single piece, and hence without any expensive and potentially unreliable joints; it has a diameter of about $6 \mathrm{~m}$, thickness varying between 0.3 and $3.2 \mathrm{~mm}$, and a total mass of around $20 \mathrm{~kg}$.

The folding concept [9] is both simple and effective: opposite edges of the reflector are pulled towards each other by about half of their original distance, and thus the reflector becomes folded elastically, as shown in Fig. 1. Once in orbit, the tie cables that hold the reflector in its packaged configuration are cut, and the reflector deploys by releasing its stored strain energy.

Intuitively, a "floppy" structure is more likely to survive this process undamaged than a "stiff" structure, but reflector structures of 


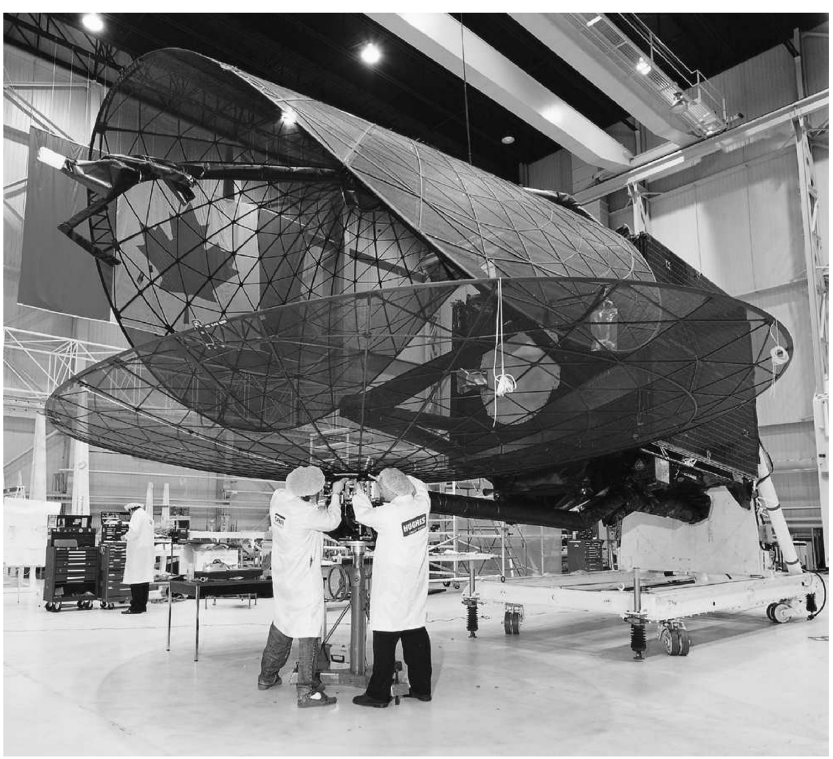

Fig. 1 MSAT-2 spacecraft during ground testing with two spring back reflectors, one deployed and one folded. Courtesy: Communications Research Centre (CRC), Industry Canada.

low stiffness have poor shape accuracy [10] and are also prone to becoming dynamically coupled with the attitude control of the spacecraft. Both of these limitations would severely restrict the potential range of applications.

This paper proposes a modification of the spring back reflector concept, based on the idea of adding a thin-walled stiffening element around the edge of the dish. This element significantly increases the overall stiffness of the dish in the deployed configuration, and yet it is engineered in such a way that the stiffened dish can still be folded elastically. A detailed description of the stiffening concept together with the subtleties that are required to make this approach work are presented.

The paper is presented in seven sections plus an appendix. Following the present Introduction, Secs. II and III explain the concept of exploiting geometrically nonlinear effects through which an elastic structure becomes softer while it is folded. The concept is illustrated by comparing two different designs for a coilable cantilever beam. It is shown that a beam whose cross section can be flattened before coiling can be several orders of magnitude stiffer than a standard, rigid cross-section beam of equal mass. Section IV presents a straightforward extension of Rayleigh's classical work on the vibration of thin spherical shells, leading to an approximate, analytical expression for the natural frequencies of vibration of a spherical cap with an edge stiffener. The fundamental natural frequency ratio, which is a measure of the stiffness increase achieved divided by the mass penalty, can thus be estimated. The range of applicability of this result is discussed and its accuracy is assessed. With this background, Sec. V carries out a detailed study of seven different configurations of the edge stiffener, for the particular case of a small scale dish. For each configuration, detailed finite-element simulations of the folding process are performed in order to estimate the deployed fundamental natural frequency, initial stiffness of the reflector, packaging force characteristics, and the maximum stresses in the packaged configuration. For some selected configurations, the sensitivity of these results to two key design parameters of the stiffener is determined. This work leads to the choice of a specific configuration of the stiffener, consisting of a conical-section ring that is attached to the rim of the reflector, but left disconnected at four locations. In practice, the edge stiffener would be made as an integral part of the structure, and four slits would be cut along the connection between the two surfaces. Section VI presents an optimized version of the configuration selected in the previous section. Section VII concludes the paper. The Appendix presents a derivation of the spherical equivalent to a dish of parabolic shape.

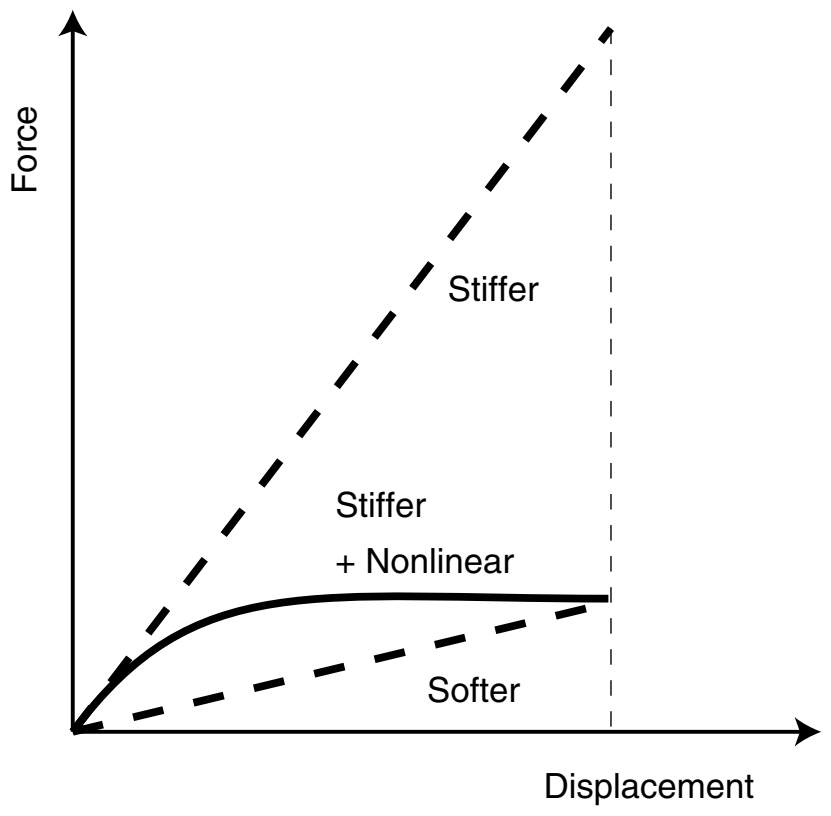

Fig. 2 Schematic of folding force-displacement relationships for linear and nonlinear structures.

\section{Structures that Become Softer During Folding}

Consider a general deployable structure made of linear-elastic material, which is assumed to deform according to a linear forcedisplacement relationship when it is folded. Here, the displacement variable is a parameter that describes the configuration of the structure, and the force variable is the corresponding value of the external action(s) required to keep the structure in static equilibrium in each particular configuration. Now, consider a modified design of this structure which is 1) stiffer than the initial design and 2) follows the same deformation mode when it is folded as the initial design. The force-displacement relationships of the two designs are schematically shown by the two dashed straight lines labeled "softer" and "stiffer" in Fig. 2.

Because the "stiffness" of the structure has been increased, the slope of the force-displacement relationship will be higher. Furthermore, because the final value of the displacement parameter in the folded configuration is the same, the total amount of strain energy required to fold the structure will increase proportionally with its stiffness.

Now, if it is further assumed that the folding of the structure can be modeled as a process that involves purely beam-bending deformation, two extreme ways of achieving the stiffness increase are 1) to increase the width of the bending elements, in which case the stiffness increase is directly proportional to the width increase, or 2) to increase their thickness, in which case the stiffness increases with the cube of the thickness. In terms of mass efficiency, case 2) is clearly superior to 1) but the maximum bending stress in the folded configuration will also increase in proportion to the thickness. In fact, neither scheme is ideal for lightweight deployable structures.

In deployable structures of simple shape it is already well known that the best way of designing for high deployed stiffness and low maximum stress in the folded configuration is to exploit geometrically nonlinear behavior. This can be achieved, for example, by allowing a thin-walled, transversally curved strip to become flatter as it begins to fold. Further details are given in the next section.

\section{Coilable Beams}

In this section we consider the simple problem-simple in the sense that it admits a full analytical solution — of designing a straight, uniform metallic beam that can be coiled uniformly, without yielding. For a given radius of coiling $c$, we compare the stiffest possible design that can be achieved for a beam with rectangular 


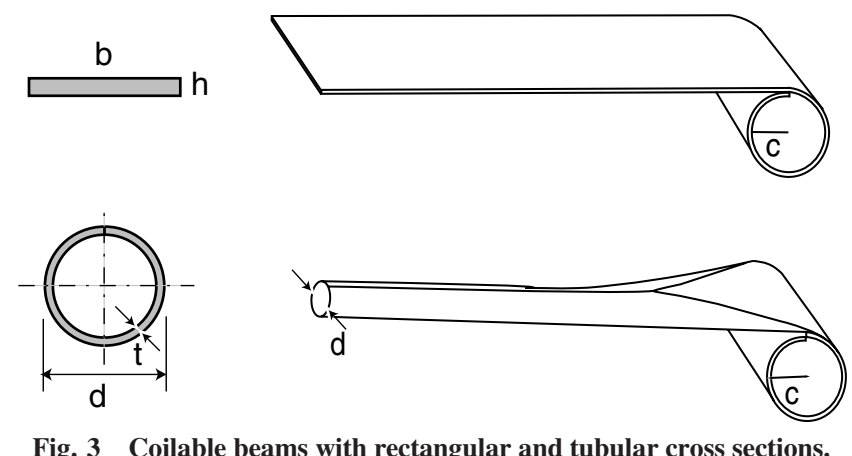

Fig. 3 Coilable beams with rectangular and tubular cross sections.

cross section with an alternative design based on a slit circular tube, as in Fig. 3. This alternative design allows the beam cross section to be flattened before being coiled. Both the total cross-sectional area and the material of the beam, and hence the mass of both designs, are the same.

In the rectangular cross-section beam of breadth $b$ and height $h$, the maximum stress in the coiled configuration is

$$
\sigma=\frac{E h}{2 c}
$$

If the maximum allowable value for $\sigma$ is the yield stress $\sigma_{y}$, then the maximum value of $h$ is

$$
\bar{h}=2 c \sigma_{y} / E
$$

and the bending stiffness of this maximally stiff rectangular-section beam-neglecting the modulus-is

$$
I_{R}=b \bar{h}^{3} / 12=\frac{2}{3} b c^{3}\left(\frac{\sigma_{y}}{E}\right)^{3}
$$

Next, consider a tubular beam whose cross section has diameter $d$, thickness $t$, and is split at the top. To make its cross-sectional area, and hence its mass, equal to that of the previous beam we set

$$
b \bar{h}=\pi d t
$$

and so, solving for $d$ and substituting Eq. (2),

$$
d=2 b c \sigma_{y} / \pi E t
$$

The value of $t$ can be determined by requiring that this beam be on the point of yielding (according to the Tresca yield condition) when its cross section is flattened and the beam is longitudinally coiled. Rimrott [1] has shown that this condition requires

$$
\frac{d}{t}=\frac{E}{\sigma_{y}} \frac{(2 c / d)+v}{\left(1-v^{2}\right)(2 c / d)}
$$

Substituting Eq. (5) into Eq. (6), one obtains a quadratic equation in $t$, which has the solution

$$
t=\left[\sqrt{\left(\frac{v}{2 \pi}\right)^{2}+\frac{2\left(1-v^{2}\right)}{\pi} \frac{c}{b}}-\frac{v}{2 \pi}\right] \frac{\sigma_{y}}{E} b
$$

When this beam is uncoiled, its bending stiffness-again neglecting the Young's modulus-is

$$
I_{T} \approx \frac{\pi d^{3} t}{8}
$$

where $d$ and $t$ can be obtained from Eqs. (5) and (7).

To compare the static stiffness of these two beams, consider the ratio $I_{T} / I_{R}$, which can be shown to have the expression

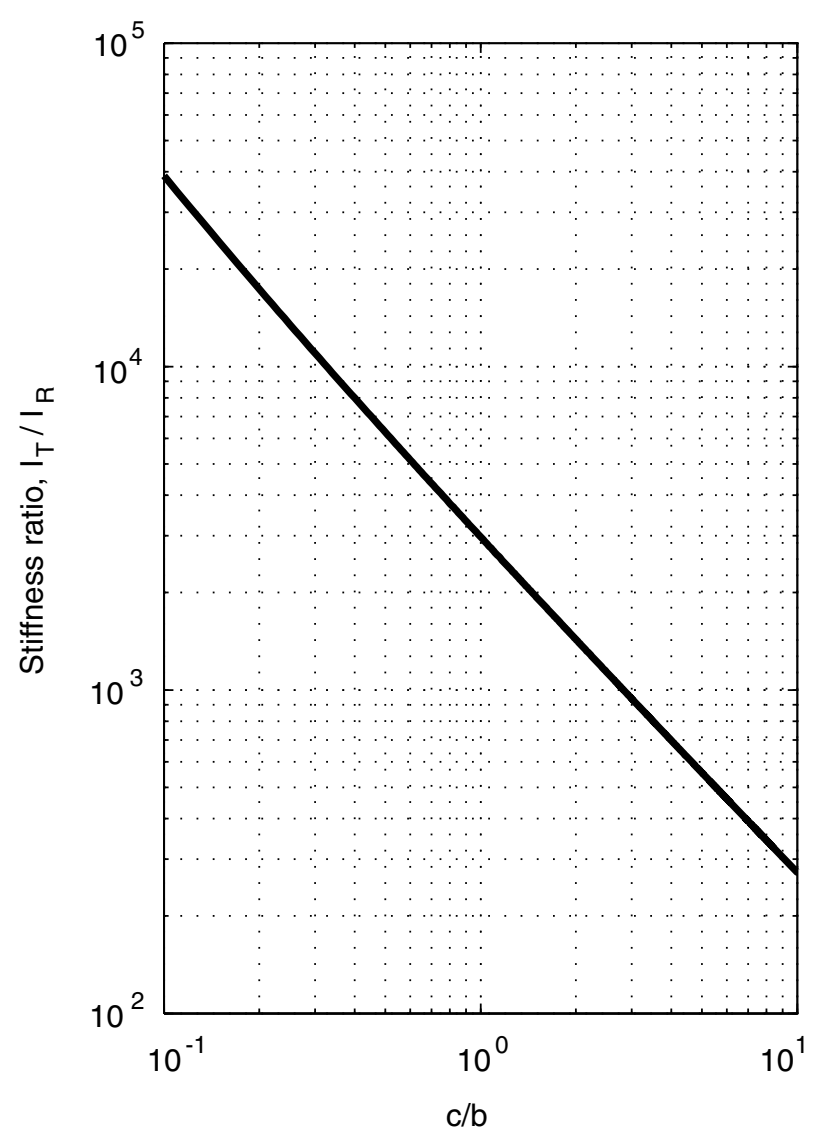

Fig. 4 Variation of flexural stiffness of a maximally stiff tubular beam with respect to the stiffness of a rectangular cross-section beam, for $E / \sigma_{y}=100$.

$$
\frac{I_{T}}{I_{R}}=\frac{3}{2 \pi^{2}}\left(\frac{E}{\sigma_{y}}\right)^{2} /\left[\sqrt{\left(\frac{v}{2 \pi}\right)^{2}+\frac{2\left(1-v^{2}\right)}{\pi} \frac{c}{b}}-\frac{v}{2 \pi}\right]^{2}
$$

For equal mass beams the ratio $I_{T} / I_{R}$ is inversely proportional to the radius of coiling $c$, divided by the breadth of the original rectangular section $b$, and is directly proportional to the squared ratio of the modulus divided by the yield stress. Note that this latter proportion $\left(E / \sigma_{y}\right)$ has typical values of 50-200 for most structural materials.

Equation (9) has been plotted for $E / \sigma_{y}=100$ in Fig. 4. The plot shows that the stiffness ratio is in excess of 200 , even for loosely coiled beams, and much larger for more tightly coiled beams.

This example has shown that a beam with a rectangular cross section is much less stiff than a beam of equal mass whose tubular cross section can be flattened before coiling. Noncircular cross sections could provide even stiffer designs, but the example presented above has demonstrated the potential advantages of designing thin-walled foldable structures with stiffening elements that increase the stiffness of the deployed structure, but can be elastically collapsed before folding.

\section{Spherical Caps}

The stiffness of a parabolic dish can be increased in many different ways. An obvious way is increasing the thickness of the dish uniformly; other options are adding a solid ring beam around the rim of the dish, or adding a series of radial/hoop ribs, etc. We are interested in choosing a solution that requires only a very small increase in mass to provide a large increase in stiffness, and the most effective way of doing this is by adding a thin-walled stiffener around the rim.

We show this by investigating how the fundamental natural frequency of an open spherical cap, which is a close approximation to 


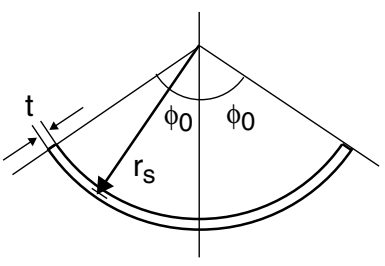

a)

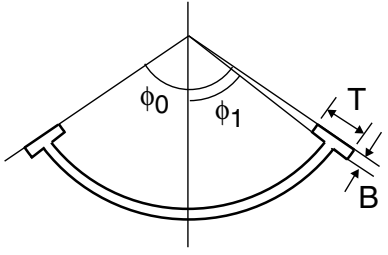

b)
Fig. 5 Cross sections of a) unstiffened and b) stiffened spherical caps.

a thin-walled, parabolic shell reflector (see the Appendix for details), varies when a ring beam is monolithically attached along the rim. We will show that the stiffness of the fundamental mode of vibration of the spherical cap increases with the cube of the depth of this edge stiffener and, neglecting the mass of the stiffener, the corresponding natural frequency increases with the 1.5 power of the depth of the stiffener.

Consider a thin-walled, isotropic and homogeneous spherical cap, with midsurface radius $r_{s}$, uniform thickness $t$, and subtending an angle $2 \phi_{0}$ as shown in Fig. 5 a.

A good estimate of the fundamental natural frequency of this shell structure can be obtained [11] by assuming an inextensional mode of vibration of dimensionless amplitude $A_{i}$, with $i(=2,3, \ldots)$ circumferential waves. Considering a polar coordinate system $r, \theta$, $\phi$, the radial components of motion, denoted by the subscript $r$, have the expression

$$
w_{r}=A_{i} r_{s}(i+\cos \phi) \tan ^{i} \frac{\phi}{2} \sin i \theta
$$

The corresponding hoop and meridional components are obtained by imposing the condition that no extension takes place.

Rayleigh [11] derived the following expressions for the strain energy $U$ and kinetic energy $V$, associated with harmonic vibration at angular frequency $\omega_{i}$

$$
\begin{gathered}
U\left(i, \phi_{0}\right)=2 \pi\left(i^{3}-i\right)^{2}(1-v) D g_{1}\left(i, \phi_{0}\right) A_{i}^{2} \sin ^{2} \omega_{i} t \\
V\left(i, \phi_{0}\right)=\frac{\pi}{2} \rho t r_{s}^{4} g_{2}\left(i, \phi_{0}\right) \omega_{i}^{2} A_{i}^{2} \cos ^{2} \omega_{i} t \\
g_{1}(i, \phi)=\frac{1}{8}\left[\frac{\left(\tan \frac{\phi}{2}\right)^{2 i-2}}{i-1}+\frac{2\left(\tan \frac{\phi}{2}\right)^{2 i}}{i}+\frac{\left(\tan \frac{\phi}{2}\right)^{2 i+2}}{i+1}\right] \\
g_{2}(i, \phi)=\int_{0}^{\phi}\left(\tan \frac{\phi}{2}\right)^{2 i}\left[(i+\cos \phi)^{2}+2(\sin \phi)^{2}\right] \sin \phi \mathrm{d} \phi
\end{gathered}
$$

The form of the equations presented above is largely due to Blevins [12]. Note that $D$ is the flexural stiffness of the shell, that is,

$$
D=\frac{E t^{3}}{12\left(1-v^{2}\right)}
$$

The corresponding natural frequencies of vibration, obtained by equating the maximum strain energy (setting $\sin \omega_{i} t=1$ ) to the maximum kinetic energy (setting $\cos \omega_{i} t=1$ ) in each mode are then

$$
f_{i}=\frac{\omega_{i}}{2 \pi}=\frac{i^{3}-i}{2 \pi} \sqrt{\frac{E / \rho}{3(1+\nu)}} \sqrt{\frac{g_{1}(i, \phi)}{g_{2}(i, \phi)}} \frac{t}{r_{s}^{2}} \quad \text { for } i=2,3,4, \ldots
$$

For a preliminary estimate of the fundamental natural frequency of a parabolic reflector we assume $r_{s}=3100 \mathrm{~mm}, t=1 \mathrm{~mm}$, $\phi_{0}=48 \mathrm{deg}, E=40,000 \mathrm{~N} / \mathrm{mm}^{2}, v=0.3$, and $\rho=980 \mathrm{~kg} / \mathrm{m}^{3}$. The fundamental mode has $g_{1}=0.030$ and $g_{2}=0.033$, and is consequently $0.305 \mathrm{~Hz}$.

Next, consider adding a light, solid ring beam around the rim of the shell, of cross-sectional dimensions $B$ by $T$, as shown in Fig. 5b. Note that $B$ is measured on the midsurface of the spherical shell. It will be assumed that the natural modes of the resulting shell, consisting of a spherical cap clamped to a truncated cone, can be approximated by Eq. (10).

To estimate the natural frequencies of this stiffened shell we need to determine the strain energy associated with the chosen vibration mode; the kinetic energy is still given by Eq. (12), as the ring beam has been assumed to be light.

Because the assumed vibration modes are unchanged, the strain energy of the stiffened shell is obtained by adding the strain energy of the original shell, given by Eq. (11), and which will now be denoted by $U_{\text {sphere }}$, to the strain energy of the ring, $U_{\text {ring }}$. The strain energy of the ring beam can be obtained approximately by considering the difference between a spherical cap of thickness $T$ that subtends an angle $\phi_{0}$ and a spherical cap, also of thickness $T$, that subtends the smaller angle

$$
\phi_{1}=\phi_{0}-B / r_{s}
$$

Hence we can write

$$
U_{\text {ring }}(i)=U\left(i, \phi_{0}\right)-U\left(i, \phi_{1}\right)
$$

Equation (18) could be calculated using $U$ given by Eq. (11) but, because the width of the edge stiffener is too small for the plate flexural stiffness $D$ to be activated, it will be replaced with the bending stiffness per unit width, $E T^{3} / 12$. Hence, we obtain

$$
U_{\text {ring }}(i)=\frac{\pi\left(i^{3}-i\right)^{2}(1-v) E T^{3}\left[g_{1}\left(i, \phi_{0}\right)-g_{1}\left(i, \phi_{1}\right)\right] A_{i}^{2} \sin ^{2} \omega_{i} t}{6}
$$

Thus, the frequency of mode $(i-1)$ of the stiffened shell, again obtained by equating maximum strain and kinetic energies, is

$$
\begin{aligned}
& f_{i}^{\prime}=\frac{i^{3}-i}{2 \pi} \sqrt{\frac{E}{\rho}} \sqrt{\frac{g_{1}\left(i, \phi_{0}\right)+\left(1-v^{2}\right)\left[g_{1}\left(i, \phi_{0}\right)-g_{1}\left(i, \phi_{1}\right)\right]\left(\frac{T}{t}\right)^{3}}{3(1+v) g_{2}\left(i, \phi_{0}\right)}} \frac{t}{r_{s}^{2}} \\
& \quad \text { for } i=2,3,4, \ldots
\end{aligned}
$$

We can determine the effectiveness of adding an edge stiffener to a spherical cap by considering the ratio between two corresponding frequencies, and, in particular, the ratio between the fundamental frequencies. The stiffness ratio is given by the square of the frequency ratios.

Dividing Eq. (20) by Eq. (16) and simplifying, or alternatively taking directly the square root of the strain energy ratio, because the kinetic energies cancel out, we obtain

$$
\frac{f_{i}^{\prime}}{f_{i}}=\sqrt{1+\left(1-v^{2}\right) \frac{g_{1}\left(i, \phi_{0}\right)-g_{1}\left(i, \phi_{1}\right)}{g_{1}\left(i, \phi_{0}\right)}\left(\frac{T}{t}\right)^{3}}
$$

This equation shows that for any value of $i$ the frequency ratio is proportional to the power 1.5 of the thickness of the stiffener divided by the thickness of the shell, whereas the stiffness ratio is dependent on the third power of this thickness ratio. The dependence with the breadth of the stiffener $B$ is hidden behind the variation of $g_{1}\left(\phi_{1}\right)$ and is more readily capture by plotting $f_{2}^{\prime} / f_{2}$ for different widths of the stiffener; see Fig. 6.

From the figure, note that the most efficient way of increasing the fundamental frequency of the reflector is to increase the depth of the stiffener $T$. Also note that the frequency increases that can be achieved are potentially very significant. For example, the addition of a $1 \mathrm{~mm}$ wide and $40 \mathrm{~mm}$ thick stiffener to the edge of the reflector described above (with $B / t=1$ ) would increase its fundamental natural frequency from 0.305 to $2.382 \mathrm{~Hz}$. More refined estimates of these frequencies, obtained from ABAQUS [13] finite-element simulations are 0.301 and $2.840 \mathrm{~Hz}$, respectively.

Note, however, that very deep stiffeners, with $T$ of the order of $\sqrt{r_{s} t}$ or greater, will also have their own vibration modes and, while 


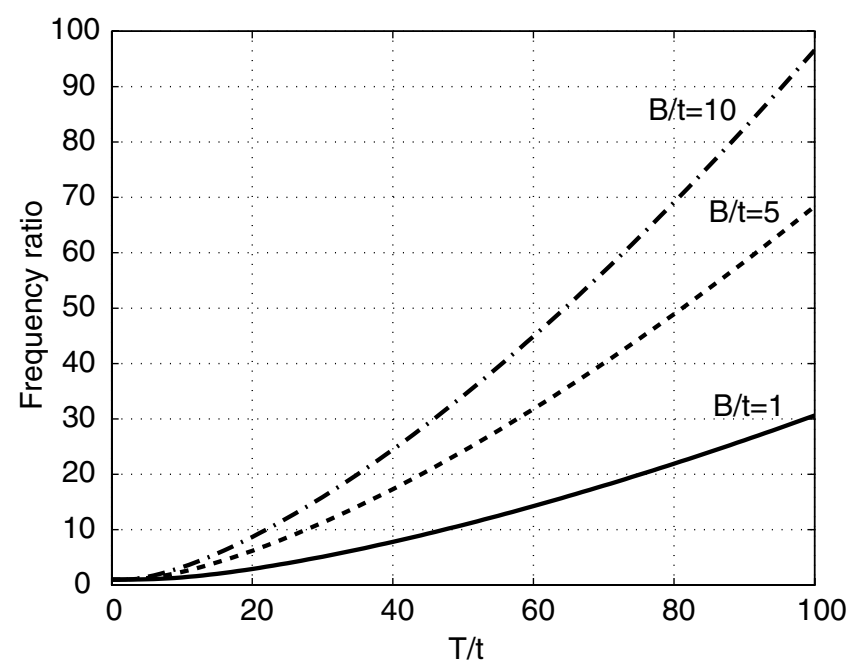

Fig. 6 Variation of corresponding natural frequencies with crosssectional dimensions of stiffener.

Eq. (20) may still be reasonably accurate in predicting the frequency of the main mode of the dish, the existence of lower frequency modes for the edge stiffener should be expected in such cases. Recall that for an axisymmetric shell subject to an edge disturbance $2.5 \sqrt{r_{s} t}$ is the distance at which the amplitude of the disturbance decays to about $4 \%$.

Also note that, in showing the potential effectiveness of providing an edge stiffener for a thin-shell reflector, we have not considered one essential requirement, that the stiffened reflector should not break when it is folded.

\section{Stiffened Reflector Dishes}

It has been shown that the addition of a stiffener around the rim of a thin dish greatly increases the stiffness of the structure and hence its fundamental natural frequency. However, a key problem with introducing such a continuous stiffener is that it makes the structure so stiff that it can no longer be folded elastically.

Following the general reasoning presented in Sec. II, this problem will be addressed by introducing either a small number of radial cuts in the stiffener or circumferential slits between the stiffener and the rim of the dish, which have the purpose of facilitating the folding of the structure without significantly reducing its stiffness in the deployed configuration. A list of the different configurations that have been considered is presented in Table 1 , where the diameters $d_{1}$ and $d_{2}$ are defined in Fig. 7a. A small scale model of the spring back reflector, approximately $1 / 10$ th scale of a full-size structure, has been used to study the performance of these different stiffened configurations.

A particular type of behavior that is obtained for some cases is that the cuts/slits allow the stiffener to "snap through" while the reflector is being folded, thus decreasing the force required to fold the reflector and also reducing the peak stresses in the structure. It should be noted that Greschik [4] also introduced cuts in a thin-walled reflector structure to allow the formation of localized folds in the surface, but his analysis of the stresses that occur during folding was purely geometric.

Table 1 Configurations of a stiffened reflector

\begin{tabular}{lcc}
\hline \hline & Name & Description \\
\hline Reference configurations & $\mathrm{O}$ & Original configuration (unstiffened) \\
& OS & Continuous stiffener (no cuts, no slits) \\
Stiffener with cuts & A0 & Cuts along diameter $d_{1}$ \\
& A90 & Cuts along diameter $d_{2}$ \\
& B & Cuts along diameters $d_{1}$ and $d_{2}$ \\
Stiffener with slits & C0 & Slits at ends of diameter $d_{1}$ \\
& C90 & Slits at ends of diameter $d_{2}$ \\
& D & Slits at ends of diameters $d_{1}$ and $d_{2}$ \\
\hline \hline
\end{tabular}

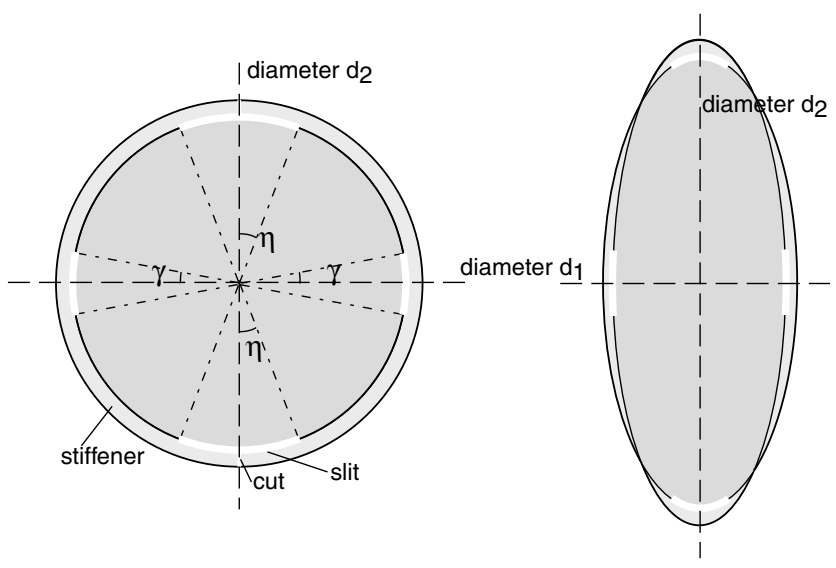

a) Plan views (deployed and folded)

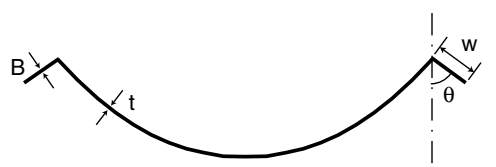

b) Cross section

Fig. 7 Design parameters of the stiffener.

\section{A. Modeling Details}

The particular structure that has been analyzed has a uniform thickness of $t=1 \mathrm{~mm}$ and forms an axisymmetric paraboloidal equation $z=\left(x^{2}+y^{2}\right) / 4 F$, where $F$ is the focal length. The focus to diameter ratio is $\frac{F}{d}=0.296$; the diameter of the aperture is $d=452 \mathrm{~mm}$. The material properties were based on a thermoplastic material with the trade name of Vivak [14], from which several experimental dishes were manufactured [15]. The properties of Vivak are $E=2 \mathrm{GPa}, \rho=1270 \mathrm{~kg} / \mathrm{m}^{3}$, and $\nu=0.3$.

The edge stiffener was modeled as a conical surface which was fully connected to the rim of the parabolic dish. The slits between the dish and the stiffener were modeled by defining two separate sets of geometrically coincident nodes on either side of each slit. The cuts in the stiffener were modeled by simply leaving the respective edges of the stiffener free from any symmetry boundary conditions. Moving boundary conditions were applied to the rim of the reflector, to impose a total displacement of about $d / 2$. The mesh density was 1000 elements per quarter mesh.

A typical analysis consists of two steps: the first, a linear eigenvalue analysis to extract the natural frequencies and eigenmodes of the reflector in its deployed configuration, and the second step, a geometrically nonlinear simulation of the folding of the reflector. The fundamental natural frequency is used as a measure of the reflector's stiffness in the deployed configuration, while the initial slope of the force-displacement relationship $k$, which defines the resistance to folding, the maximum stress $\sigma_{\max }$, the maximum force that needs to be applied to fold the reflector $F_{\max }$, and the final value of the force required to hold the reflector in its packaged configuration $F_{\text {final }}$, were all extracted from the latter step.

\section{B. Finite Element Model Verification}

First, the fundamental frequency of the unstiffened reflector obtained from the finite element (FE) model was compared to an approximate analytical estimate based on Eq. (16).

The spherical equivalent to the structure that is being analyzed, see the derivation in the Appendix, has a radius $r_{s}=316 \mathrm{~mm}$ and subtends an angle $\phi_{0}=45.7 \mathrm{deg}$. Substituting these values and the properties of Vivak into Eq. (16) gives $6.28 \mathrm{~Hz}$, compared to $6 \mathrm{~Hz}$ for the FE model of configuration $\mathrm{O}$. The difference between the two values is $4.7 \%$ and can be attributed to the fact that the analytical estimate is for a spherical, not a parabolic shell. The FE estimate for a spherical cap is exactly $6.28 \mathrm{~Hz}$.

Next, it was verified that the quarter models set up in ABAQUS yield similar results to the corresponding full models of the structure. 

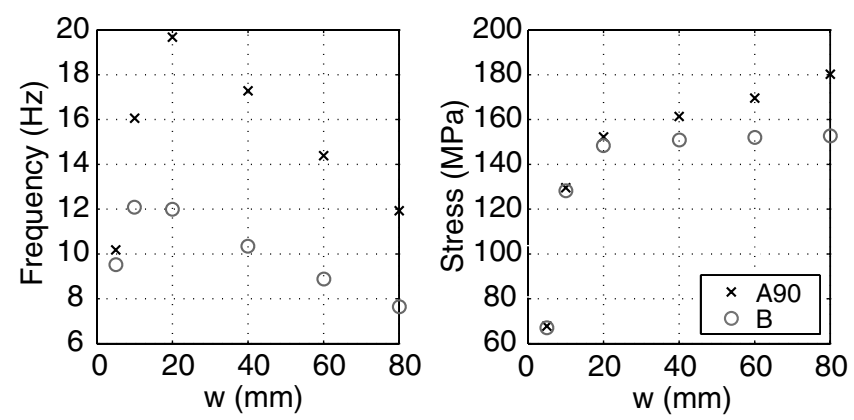

a)

b)

Fig. 8 Variation of fundamental deployed frequency and maximum packaged stress with stiffener width, for $\boldsymbol{\theta}=\mathbf{5 0} \mathbf{d e g}$.

The force-displacement curves for configuration A90 (parameters: $w=22 \mathrm{~mm}, \theta=50 \mathrm{deg}$, and $t=1 \mathrm{~mm}$ ) had negligible discrepancies while for configuration $\mathrm{D}$ (parameters: $w=10 \mathrm{~mm}$, $\theta=50 \mathrm{deg}, \eta=12 \mathrm{deg}, \gamma=4 \mathrm{deg}, t=1 \mathrm{~mm})$, the quarter model reaches a $3 \%$ higher peak force and predicts a slightly lower final force. There was also no discernible difference between full models with mirror symmetric meshes or rotated meshes.

Finally, note that the fundamental frequency of the configuration D quarter model considered above is $19.5 \mathrm{~Hz}$ while that of the full model is $19.6 \mathrm{~Hz}$, a $0.5 \%$ discrepancy.

\section{Stiffener Design Parameters}

The design parameters for the edge stiffener are the following (see Fig. 7):

1) the width $w$ and thickness of the stiffener $B$;

2) the apex angle $\theta$ of the conical surface;

3 ) the number of cuts, the number of slits, and their location;

4) the angles subtended by the slits $\gamma$ and $\eta$,

where it should be noted that, on the basis of preliminary experimentation on simple physical models, the range of options for the number and location of the cuts/slits is restricted to those listed in Table 1. Also, it was decided that the thickness of the stiffener would be set equal to that of the main shell, that is, $B=t$, for simplicity.

To obtain an indication of the effectiveness of each design parameter in increasing the fundamental natural frequency, while also evaluating the effect on the maximum stress in the folded reflector, a series of preliminary studies was carried out on configurations A90, B, and D only. In each study, only one design parameter was modified, starting from a common initial design.

First, the effect of increasing the stiffener width $w$ was considered, assuming the stiffener to be a flat horizontal plate (i.e., a cone with $\theta=90 \mathrm{deg}$ ) with either two or four cuts. Figure 8a shows that the frequency increases rapidly for $w<20 \mathrm{~mm}$ for configuration A90 and $w<10 \mathrm{~mm}$ for configuration $\mathrm{B}$, but both decrease for $w>20 \mathrm{~mm}$.

This is because the frequency is dependent on the ratio between modal mass and stiffness and, as $w$ increases, so does the mass. Beyond a certain limit, the mass penalty associated with increasing $w$ outweighs the increase of stiffness provided. This result is somewhat different from the power law relationship in Eq. (21), which assumed the added mass to be negligible, the stiffener to be perpendicular to the shell surface, and no cuts.

The effect of $w$ on the maximum stress in the packaged reflector is plotted in Fig. 8b. Increasing the width of the stiffener increases the stress, initially at a faster rate.

Second, the effect of varying the angle of the stiffener was considered, for the cases of either two or four cuts. The results are shown in Fig. 9. Both the frequency and the stress variations are roughly bilinear; the maximum frequency occurs around $\theta=80 \mathrm{deg}$, which is close to the stiffener being a flat plate. This increase of stiffness is coupled with the penalty of increased maximum stress in the packaged configuration (Fig. 9b).
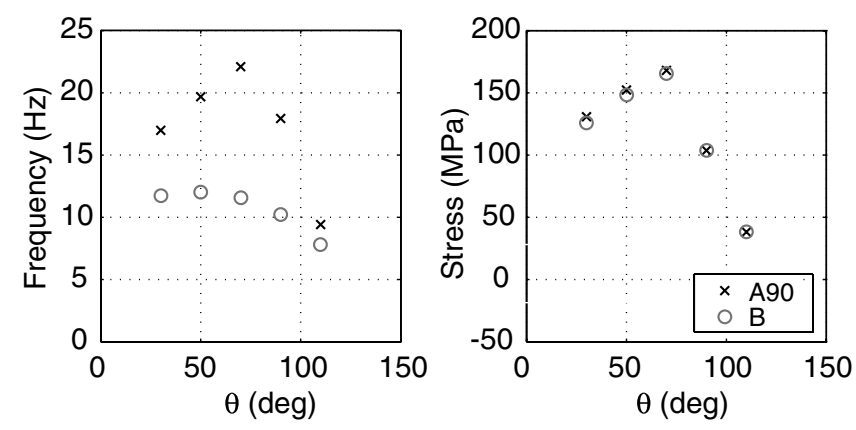

a)

b)

Fig. 9 Variation of fundamental deployed frequency and maximum packaged stress with stiffener angle, for $w=20 \mathrm{~mm}$.

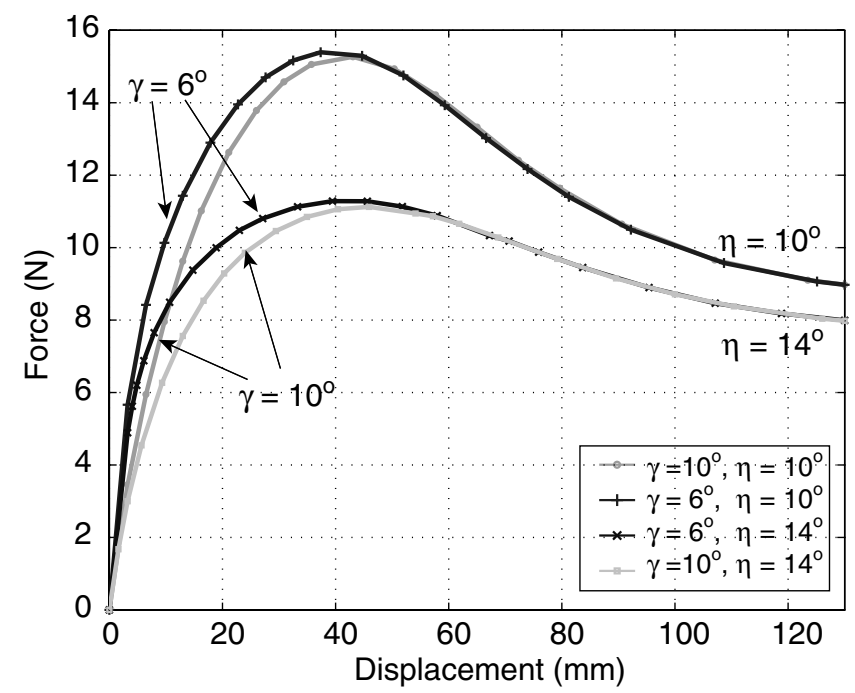

Fig. 10 Variation of folding force-displacement relationship with stiffener slit angles, for $w=20 \mathrm{~mm}$ and $\theta=50 \mathrm{deg}$.

The above results show that, although effective in increasing the deployed frequency, the introduction of radial cuts in the stiffener leads to high peak stresses. These high stresses arise from having localized the bending of the main dish near the apex of the cuts, and hence the maximum stress is relatively insensitive to the addition of radial cuts along diameter $d_{1}$.

This sharp localization in the bending of the surface is avoided in configuration D. This is because the slits allow a larger area of the dish to bend, rather than concentrating the curvature change along a narrow hingelike region of the dish. Two pairs of diametrically opposite slits were considered in the present study; the resulting loaddisplacement plots are shown in Fig. 10.

The pair of slits at the end of diameter $d_{2}$ serve the purpose of allowing larger portions of the dish to deform rather than localizing curvatures (Fig. 13) and so reduce the peak strains in the folded dish. They also control the final force required to keep the reflector packaged; increasing the slit angle $\eta$ reduces these forces. On the other hand, the pair of slits at the end of diameter $d_{1}$ allow the stiffener to buckle during packaging and hence affect the initial stiffness of the reflector.

\section{Packaging}

An analysis of the packaging force-displacement relationship of the reflector provides significant insight into the behavior of the structure and is also useful in making comparisons between different designs of the edge stiffener. The reflector structure is folded about diameter $d_{2}$ which is equivalent to applying two diametrically opposing forces along diameter $d_{1}$, causing it to fold in a way similar to Fig. 1. 


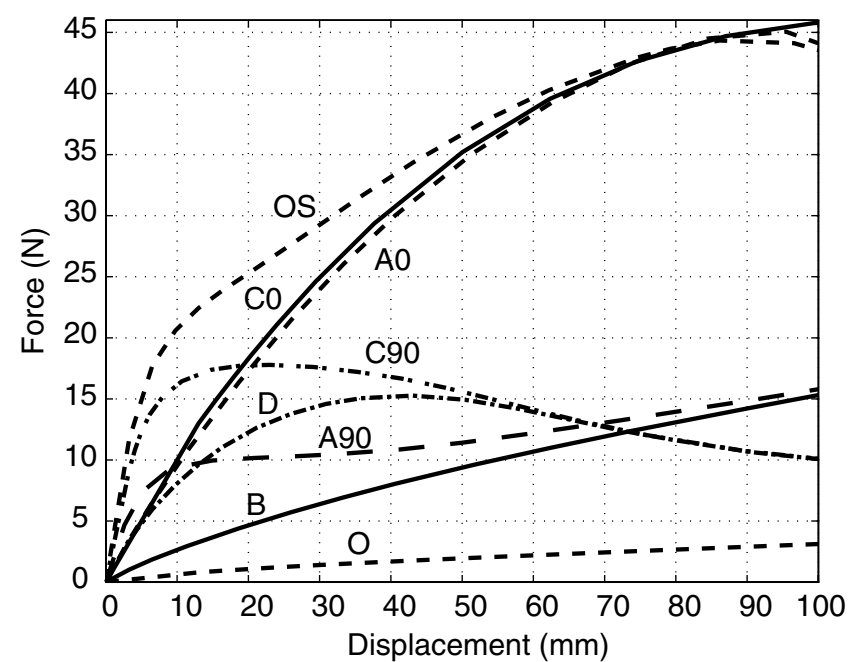

Fig. 11 Force-displacement plots for eight different designs.

This relationship has been computed for the eight different design configurations listed in Table 1; see Fig. 11. Note that the displacement plotted in Fig. 11 is that of one loaded point; the point diametrically opposite moves by an equal but opposite amount. In all cases, the stiffener width is $w=20 \mathrm{~mm}$, its angle $\theta=50 \mathrm{deg}$, and the slit angles where applicable are equal, $\eta=\gamma=10 \mathrm{deg}$.

The unstiffened reflector, $\mathrm{O}$, and the reflector with a continuous stiffener but neither slits nor cuts, OS, that is, the configurations with minimum and maximum initial stiffness, were analyzed to obtain two reference curves. In all cases it was assumed that the behavior of the material is linear elastic, regardless of strain magnitudes.

Table 2 compares key characteristics of these different configurations. Columns $2-4$ list the ratios between the initial (i.e., tangent) stiffness in the deployed configuration $k$, the fundamental natural frequency of vibration $f$, the final packaging force $F_{\text {final }}$, and the maximum force reached during packaging $F_{\max }$ with respect to the unstiffened reflector, whose values are denoted by the subscript 0 . Column 5 lists the ratios between the final and maximum force. Column 6 lists the maximum stresses $\sigma_{\max }$ in the packaged reflector assuming the structure to be made of Vivak and yet to remain linear elastic regardless of the large stress magnitude.

It is useful to discuss the response curves by grouping them into sets that share common features, as follows:

1) Very low initial stiffness and very low packaging force: configuration $\mathrm{O}$. The unstiffened configuration has an initial stiffness of $0.06 \mathrm{~N} / \mathrm{mm}$ and a final packaged force of $3.1 \mathrm{~N}$.

2) Low initial stiffness and high packaging force: configuration $B$. The reason for the high packaging force is that the reflector folds into two almost rigid half-shells connected by an elastic hinge aligned with the two cuts. The transverse curvature in this hinge region increases with the amount of folding and its longitudinal curvature is approximately zero. Hence, after some initial nonlinearity the forcedisplacement relationship is almost linear. However, note that configuration B has the lowest initial stiffness among all stiffened configurations, despite a fivefold increase on configuration $\mathrm{O}$.

Table 2 Key characteristics of different structural configurations

\begin{tabular}{lrrrrr}
\hline \hline Config. & \multicolumn{1}{c}{$\frac{k}{k_{0}}$} & $\frac{f}{f_{0}}$ & $\frac{F_{\text {final }}}{F_{\text {fina }}}$ & $\frac{F_{\text {final }}}{F_{\max }}$ & $\sigma_{\max }, \mathrm{MPa}$ \\
\hline O & 1.0 & 1.0 & 1.0 & 1.0 & 10 \\
OS & 53.2 & 5.0 & 13.9 & 1.0 & 181 \\
A0 & 17.4 & 3.3 & 14.1 & 1.0 & 179 \\
A90 & 27.2 & 3.3 & 5.1 & 1.0 & 152 \\
B & 5.0 & 1.6 & 6.0 & 1.0 & 151 \\
C0 & 16.5 & 4.2 & 14.7 & 1.0 & 100 \\
C90 & 46.1 & 4.2 & 3.2 & 0.6 & 85 \\
D & 16.7 & 3.5 & 3.2 & 0.7 & 84 \\
\hline \hline
\end{tabular}


Table 3 Comparison of optimized configuration D

\begin{tabular}{lcccrc}
\hline \hline Config. & $\begin{array}{c}k, \\
\mathrm{~N} / \mathrm{mm}\end{array}$ & $\begin{array}{c}f, \\
\mathrm{~Hz}\end{array}$ & $\begin{array}{c}F_{\text {final }}, \\
\mathrm{N}\end{array}$ & $\begin{array}{r}\sigma_{\max }, \\
\mathrm{MPa}\end{array}$ & $\begin{array}{c}\text { Mass, } \\
\mathrm{kg}\end{array}$ \\
\hline O & 0.06 & 6.00 & 3.10 & 9.98 & 0.236 \\
Optimal D & 1.88 & 24.13 & 4.11 & 25.91 & 0.274 \\
C90 & 2.72 & 24.76 & 4.18 & 39.18 & 0.274 \\
\hline \hline
\end{tabular}

has been shown [16] that detailed redesign of these regions, by rounding the corners and/or grading the stiffness of the material, can reduce these stresses to values close to those that occur elsewhere in the reflector. This redesign is best carried out as a separate step, after determining the overall distribution of stress in the folded reflector.

An automatic mesh generation procedure was created, and the Hooke and Jeeves direct search method was used to find a series of optimal configurations. Details of this study will be published in a separate paper [17].

An optimal configuration $\mathrm{D}$, obtained for $\sigma_{\max }=26 \mathrm{MPa}$, is presented in Table 3 . The corresponding values of the design parameters are the following:

1) apex angle of stiffener $\theta=88.1 \mathrm{deg}$,

2) width of stiffener $w=20 \mathrm{~mm}$,

3) angle of slit at end of diameter $d_{1}, \gamma=6.1 \mathrm{deg}$,

4) angle of slit at end of diameter $d_{2}, \eta=17.4 \mathrm{deg}$.

Figure 12 compares the load-displacement relationship of this optimized structure (optimal D) with the original, unstiffened structure $(\mathrm{O})$ and a stiffened structure with only two slits (C90), which, however, has a maximum stress well in excess of the specified limit. This C90 configuration is identical to optimal D except for the fact that the pair of slits at the ends of diameter $d_{1}$ have been removed, that is, $\eta=17.4 \mathrm{deg}$ and $\gamma=0 \mathrm{deg}$.

More detailed information on the stress distribution in the folded structure is presented in Fig. 13. It can be observed that the highest stresses occur in the unsupported regions of the stiffener, the first region of high stress being the stiffener region adjacent to the shorter slit and the second region being the area surrounding the tip of the longer slit.

\section{Discussion and Conclusions}

The simple analytical estimates presented in Sec. IV have allowed us to quantify the increases in a thin-shell reflector's deployed stiffness, and fundamental natural frequency of vibration, that are achieved through the addition of a thin stiffener around the rim of the reflector structure. It has been shown that tenfold increases in frequency can be achieved quite easily. Hence, increasing the deployed stiffness of a thin-shell reflector structure is not difficult, but the key problem is ensuring that the stiffened structure can still be folded elastically.

Two different ways of achieving stiffer reflectors that can still be folded elastically have been explored, through the introduction of 1) cuts or 2) slits in the stiffener. The first approach, with cuts in the stiffener, tends to concentrate the deformation of the folded reflector in a narrow strip. This results in maximum stresses that are at least 15 times larger than the maximum stress in the unstiffened dish. The second approach, with circumferential slits, spreads the deformation over a wide region, whose width is largely controlled by the length of the slits at the end of diameter $d_{2}$. Hence, it has been concluded in the paper that the introduction of slits between the dish and the stiffener is the most effective way forward.

Varying the length of the two pairs of slits is a way of controlling the stiffness of the deployed reflector, the maximum disturbance force that it will withstand without starting to fold, and the maximum stress in the folded reflector. Very broadly speaking, for a stiffener of given width and thickness, shortening the slits at the end of diameter $d_{1}$ increases the stiffness, whereas shortening the slits at the end of diameter $d_{2}$ has the effect of increasing the maximum stress in the folded reflector. The magnitude of the maximum force before the reflector snaps is controlled by both sets of slits and increases when they are shorter.

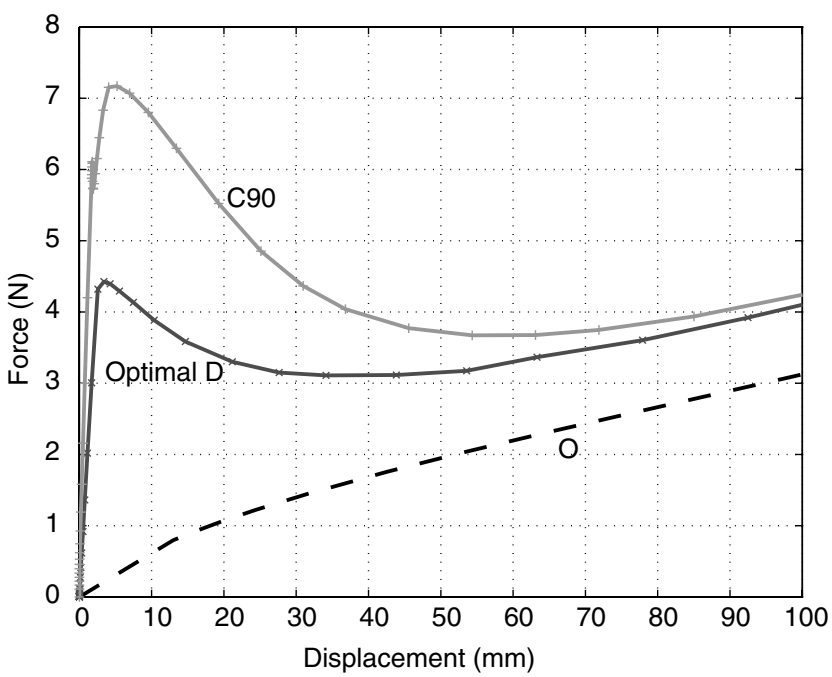

Fig. 12 Comparison of force-displacement relationship of optimized configuration $\mathrm{D}$ with original unstiffened configuration $(\mathrm{O})$ and two-slit configuration (C90).

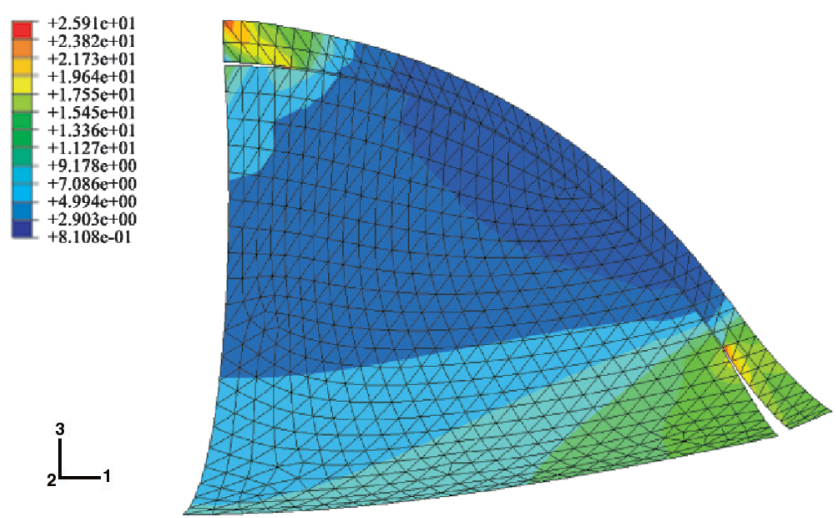

Fig. 13 Von Mises stress distribution for optimized configuration D, in MPa.

The specific case of a Vivak dish with aperture diameter of $452 \mathrm{~mm}$, thickness of $1 \mathrm{~mm}$, and focal length to diameter ratio of 0.296 has been considered. It has been shown that its stiffness in the deployed configuration increases by a factor of 53.2 when a $20 \mathrm{~mm}$ wide and $1 \mathrm{~mm}$ thick edge stiffener is continuously connected to the edge of the reflector. However, the penalty associated with this design modification is an 18-fold increase in the maximum stress in the folded reflector, which would be well in excess of the yield stress.

Hence, having assumed that Vivak would remain close to linear elastic for stresses up to $26 \mathrm{MPa}$, this value was set as a limit when searching for an optimized set of design parameters. One of the optimal configurations that was arrived at has a $20 \mathrm{~mm}$ wide by $1 \mathrm{~mm}$ thick stiffener that is nearly planar (the optimal value of the cone angle was $88.8 \mathrm{deg}$, that is, almost $90 \mathrm{deg}$ ); the slits at the end of diameter $d_{1}$ subtend a total angle of almost $35 \mathrm{deg}$ whereas the second set of slits are about one-third this length. This configuration is 31 times stiffer but is only $16 \%$ heavier than the unstiffened dish and has a maximum stress within the prescribed limit.

The force-displacement relationship for this optimized configuration is shown in Fig. 12. The significant increase in initial stiffness with respect to the unstiffened dish, and the snapping behavior of the stiffener, whereby the forces needed to package the dish suddenly decrease, are evident in the figure. Nonoptimized configurations tend not to show such a pronounced snapping behavior.

It is also insightful to make a further comparison with the corresponding nonreference configuration (C90) which was shown in Sec. V to have the highest deployed stiffness and frequency. Figure 12 shows a $42 \%$ increase in maximum "snapping" force $F_{\max }$, 


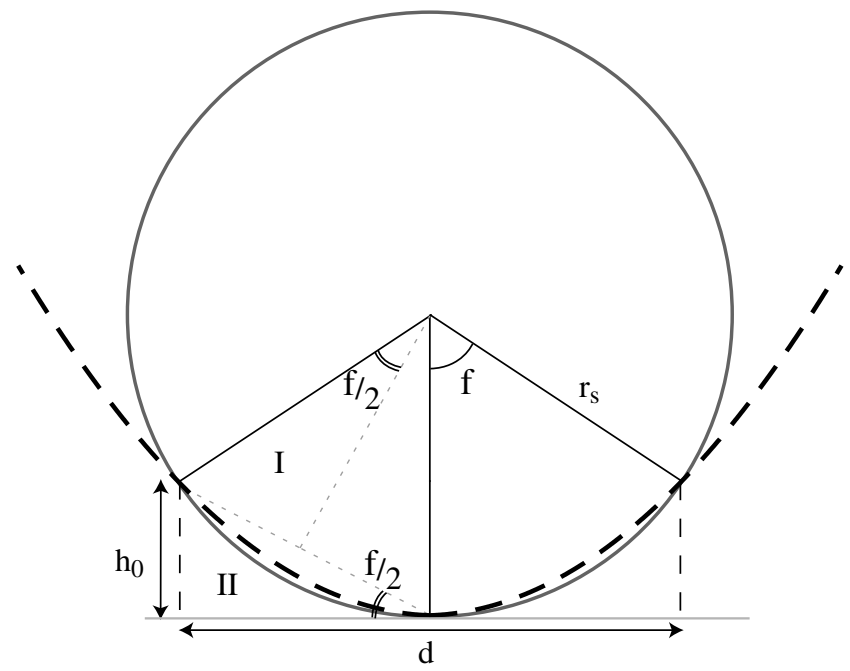

Fig. 14 Approximation of a paraboloid with a spherical cap.

but an almost identical final packaged force $F_{\text {final }}$. The ratios of $F_{\text {final }} / F_{\text {max }}$ are 0.84 and 0.60 for configurations optimal D and C90, respectively. This two-slit (C90) configuration has a $16 \%$ higher initial stiffness but a 50\% increase in packaged stress, well in excess of the specified limit. The only difference between these two configurations is the absence of slits at the end of diameter $d_{1}$; hence C90 is the limiting case of D and this demonstrates the tunability of configuration D.

In conclusion, the viability of stiffening a thin-shell deployable reflector by connecting it with a collapsible stiffener, which still allows for elastic folding and completely passive deployment, has been demonstrated. Key to this scheme are two pairs of circumferential slits which allow localized elastic buckling of the stiffener, hence significantly reducing the stiffness of the reflector when it is being packaged. The performance of the stiffener can be enhanced significantly by optimizing its design parameters. A small number (four) of adjustable parameters have been considered so far and a $70 \%$ reduction in the maximum stress in the folded reflector has been achieved, without any significant reduction in the increased stiffness of the stiffened reflector.

\section{Appendix: Derivation of Spherical Equivalent to a Parabolic Cap}

A spherical cap that approximates to a paraboloidal cap can be determined based on the assumption that both caps have the same aperture diameter $d$ and rim height $h_{0}$. The spherical cap is fully described by the radius $r_{s}$ and the angle subtended $\phi$. From triangles I and II in Fig. 14,

$$
\sin \frac{\phi}{2}=\frac{\sqrt{(d / 2)^{2}+h_{0}^{2}}}{2 r_{s}}=\frac{h_{0}}{\sqrt{(d / 2)^{2}+h_{0}^{2}}}
$$

Solving for $r_{s}$ and noting that $h_{0}=d^{2} / 16 F$ gives

$$
\begin{aligned}
r_{s} & =\frac{1}{2}\left(\frac{d^{2}}{4 h_{0}}+h_{0}\right) \\
& =2 F+\frac{d^{2}}{32 F}
\end{aligned}
$$

whereas the subtended angle of the spherical cap is given by

$$
\begin{gathered}
\phi=\sin ^{-1}\left(\frac{d}{2 r_{s}}\right) \\
=\sin ^{-1}\left(d /\left(4 F+d^{2} / 16 F\right)\right)
\end{gathered}
$$

\section{Acknowledgment}

We thank C. R. Calladine and C. R. Steele for helpful comments.

\section{References}

[1] Rimrott, F. P. J., "Storable Tubular Extendible Member," Machine Design, Vol. 37, 1965, pp. 156-165.

[2] Aguirre, M., Bureo, R., Fuentes, M., and Rivacoba, J., "The Collapsible Tube Mast (CTM)," Second European Space Mechanisms and Tribology Symposium, European Space Agency, Meersburg, 1985, pp. $75-81$.

[3] Givois, D., Sicre, J., and Mazoyer, T., "A Low Cost Hinge for Appendices Deployment: Design Test and Applications," 9th European Space Mechanisms and Tribology Symposium, European Space Agency, Liege, Belgium, 2001, pp. 145-151.

[4] Greschik, G., "On the Practicality of a Family of Pop-up Reflectors," 9th Annual AIAA/Utah State University Conference on Small Satellites, AIAA, Washington, D.C., 1995.

[5] Romeo, R. C., Meinel, A. B., Meinel, M. P., and Chen, P. C., "UltraLightweight and Hyper-Thin Rollable Primary Mirror for Space Telescopes," UV, Optical and IR Space Telescopes and Instruments, SPIE Proceedings, Vol. 4013, edited by J. Breckinridge, SPIEInternational Society for Optical Engineering, Bellingham, WA, 2000, pp. 634-639.

[6] Tibbalds, B., Guest, S. D., and Pellegrino, S., "Inextensional Packaging of Thin Shell Slit Reflectors," Technische Mechanik, Vol. 24, No. 3-4, 2004, pp. 211-220.

[7] Seizt, P., "Spar Resolving Spat Over Antenna Work," Space News, Aug. 29-Sept. 41994.

[8] Anon., "Hughes Graphite Antennas Installed on MSAT-2 Craft," Space News, Nov. 1994.

[9] Robinson, S. A., "Simplified Spacecraft Antenna Reflector for Stowage in Confined Envelopes," Publication No. 0534110A1, filed 31 March 1993, European Patent Application filed by Hughes Aircraft Company.

[10] Rogers, C. A., Stutzman, W. L., Campbell, T. G., and Hedgepeth, J. M., "Technology Assessment and Development of Large Deployable Antennas," ASCE Journal of Aerospace Engineering, Vol. 6, No. 1, 1993, pp. 34-54.

[11] Rayleigh, J. W. S., The Theory of Sound, Vol. 1, Dover Publications, New York, 1945.

[12] Blevins, R. D., Formulas for Natural Frequency and Mode Shapes, Krieger, Malabar, FL, Chap. 12, pp. 330-334.

[13] Hibbitt, D., Karlsson, B., and Sorenson, P., ABAQUS Standard Users Manual Ver. 6.1, Hibbitt, Karlsson and Sorenson Inc., Pawtucket, RI, 1998.

[14] "Vivak Product Technical Data Sheet," http://www.sheffieldplastics. com [cited 3 Feb. 2005].

[15] Tan, L. T., and Pellegrino, S., "Stiffening Method for Thin Shell Deployable Reflectors: Part 2-Experiments, Approach," AIAA Journal (to be submitted).

[16] Tan, L. T., "Thin-Walled Elastically Foldable Reflector Structures," Ph.D. Thesis, Department of Engineering, University of Cambridge, Cambridge, U.K., 2002.

[17] Tan, L. T., and Pellegrino, S., "Stiffness Optimization for Reflector Antennas," Computers \& Structures (to be submitted). 